“C 2019 IEEE. Personal use of this material is permitted. Permission from IEEE must be obtained for all other uses, in any current or future media, including reprinting/republishing this material for advertising or promotional purposes, creating new collective works, for resale or redistribution to servers or lists, or reuse of any copyrighted component of this work in other works." 


\title{
Real-Time 3D Profiling with RGB-D Mapping in Pipelines Using Stereo Camera Vision and Structured IR Laser Ring
}

\author{
Amal Gunatilake ${ }^{1}$ (D), Lasitha Piyathilaka ${ }^{1}$ (D), Sarath Kodagoda ${ }^{1}$ (D), Stephen Barclay ${ }^{2}$, Dammika Vitanage ${ }^{2}$ \\ ${ }^{1}$ iPipes Lab, Centre for Autonomous Systems, University of Technology Sydney, Australia \\ ${ }^{2}$ Sydney Water Corporation, Parramatta, New South Wales, Australia
}

\begin{abstract}
This paper is focused on delivering a solution that can scan and reconstruct the 3D profile of a pipeline in realtime using a crawler robot. A structured infrared (IR) laser ring projector and a stereo camera system are used to generate the 3D profile of the pipe as the robot moves inside the pipe. The proposed stereo system does not require field calibrations and it is not affected by the lateral movement of the robot, hence capable of producing an accurate 3D map. The wavelength of the IR light source is chosen to be non overlapping with the visible spectrum of the color camera. Hence RGB color values of the depth can be obtained by projecting the $3 \mathrm{D}$ map into the color image frame. The proposed system is implemented in Robotic Operating System (ROS) producing real-time RGB-D maps with defects. The defect map exploit differences in ovality enabling real-time identification of structural defects such as surface corrosion in pipe infrastructure. The lab experiments showed the proposed laser profiling system can detect ovality changes of the pipe with millimeter level of accuracy and resolution.
\end{abstract}

\section{INTRODUCTION}

Underground infrastructure such as sewage pipes and water pipes undergo severe concrete [1] and metallic [2] corrosion, which considerably reduces their service life. Monitoring such degradation through predictive modeling requires reliable sensor data for high-quality predictions [3], [4], [5], [6]. However, in hostile sewer pipelines, sensors can malfunction over time [7]. In addition to monitoring physical changes of pipes, there are requirements to monitor the sensor health conditions themselves [8]. Therefore, water utilities around the world are experiencing an uphill battle for maintaining underground assets in a good condition to avoid catastrophic failures such as pipe bursts and ground collapses [9]. Further, human entry to smaller sized pipelines for visual inspections is not possible due to occupational health and safety risks. Traditionally, CCTV cameras are mounted on remotely operated robotic platforms for inspecting such pipelines, however they only provide visual cues that has limited structural information for decision making.

In recent years, 3D laser profiling with monocular vision and structured laser light has emerged as a promising technology, which can generate a 3D map of the internal surface of a pipe [10], [11], [12], [13]. The main advantage over CCTV inspection is 3D maps can easily detect and quantify structural defects on the internal pipe surface such as changes in ovality, material loss due to deterioration and or any sludge growths. However, 3D laser profiling using single camera requires extensive field calibrations and the accuracy of the 3D map is largely affected by the lateral movement of the robotic platform. Alternatively, 3D map generation is done mostly offline and therefore, it cannot be used for online as well as opportunistic decision making by using the robotic platform. If a 3D map is available in real-time, then the operation can utilize the 3D data to further investigate the presence of any defects. Further, real-time 3D maps can be used to identify any unscanned regions and improve them accordingly while in operation.

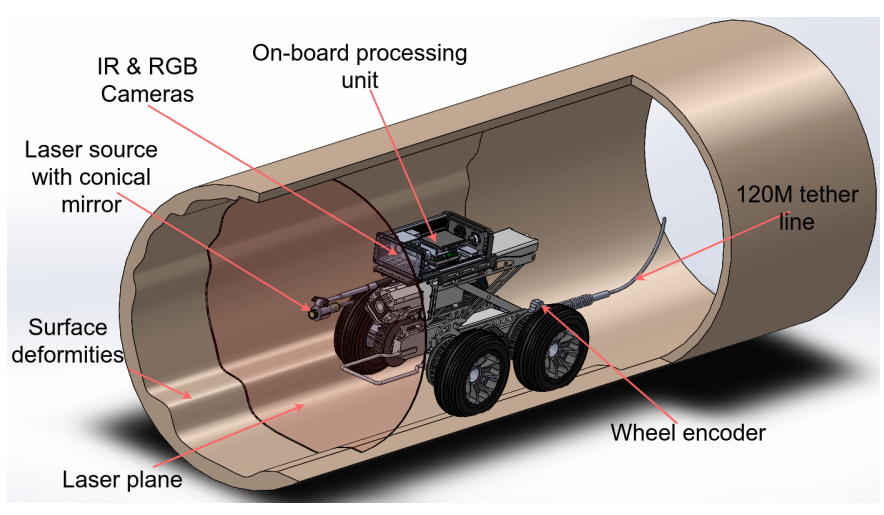

Fig. 1: Robot platform with sensors

This paper proposes a stereo vision-based system that uses IR structured light for 3D profiling inside pipes. The use of stereo cameras enables to decouple the depth measurements of the laser beam from the lateral movement of the robot hence increasing the accuracy of a 3D map. Further, use of stereo vision system does not require field calibration, and can be deployed in a wider range of pipe diameters enabling fast and convenient deployment. The proposed system has the capability to generate the $3 \mathrm{D}$ point cloud in real-time while traversing through the pipeline. The real-time 3D map of the pipeline was generated using the ROS framework, and tests were conducted to determine the effectiveness of the 3D maps. An infrared (IR) laser beam projector is proposed in this paper in lieu of traditional red colored laser beam to conserve the true color patterns of the surface on to the 3D map. An additional RGB camera is used to collect the true color data from the surface. The projected IR laser is visible in stereo IR cameras however does not interfere with color values extracted by the RGB camera. In addition, the proposed system generates a color heat map that indicates the deviation of the ovality from the original dimensions of the pipe providing a real-time defect map. 


\section{IMPLEMENTATION}

The sensing prototype was built on a robotic platform, which has the basic functionalities of inspecting pipelines. The platform is equipped with a CCTV camera including a flashlight system that can be deployed via a $120 \mathrm{~m}$ long tethered cable for connecting with the control station. The stereo camera system and the IR laser projector were built as a separate unit and mounted on the robotic platform with a dedicated computer module as shown in Fig. 1

\section{A. 3D profiling technologies}

Several technologies such as LIDAR, Time-of-flight (TOF) cameras, structured 3D cameras, and structured laser ring profiling were evaluated to identify their performance in generating an accurate 3D map of a pipeline. Among those technologies, LIDAR was less preferred for the proposed application due to low resolution in range measurements (less than $1 \mathrm{~cm}$ accuracy), and the need of slow operation for increased resolution requirements, which is not ideal for continuous pipe scanning [14]. Although most of the existing 3D cameras are inherent with TOF camera technology, it is less effective due to low grid resolution of the projected pattern. Further, when the surface is reflective the sensing of the projected pattern becomes non trivial and it can result in a considerable noise [15]. Structured light 3D cameras such as Intel Reaslsense and Microsoft Kinect motion sensor project low resolution IR patterns and therefore they are not sensitive to smaller structural variations of the sensed surface. Since this application demands millimeter $(\mathrm{mm})$ level of accuracy to detect defects on the surface, structured laser ring projection technique with the use of stereo camera vision stands as a strong choice [16], [17]. This enables to capture accurate structural information from the laser pattern while traversing the robot inside the pipelines [18], [19]. Further research work was conducted in this project to enhance the accuracy and obtain the natural colour patterns of the surface by using a stereo IR camera, a RGB camera and IR laser pattern. Using an IR laser improves the performance of extracting the colour parameters from RGB camera by filtering out the IR light.

\section{B. Hardware}

A 360 degree IR laser, which projects a beam on to the circumference of the pipe wall was used as the structured light in this setting. The laser projector was mounted at the frontend of the camera as shown in Fig. 1 to ensure the laser plane is always projected perpendicular to the robots forward motion as well as to minimize the obstruction.

Since the laser projection creates a continuous circular ring throughout the scanning surface area, even small structural changes of the surface are visible as deviations from a perfect circle. Two stereo IR cameras, each with $1280 \times 720$ resolution, have been used to extract high definition images of the laser projection. Further, an RGB camera with an IR filter, was used to extract the colour information of the pipe wall. As the chosen IR did not interfere with the RGB spectrum, the 3D point cloud of the IR laser ring generated by the stereo camera

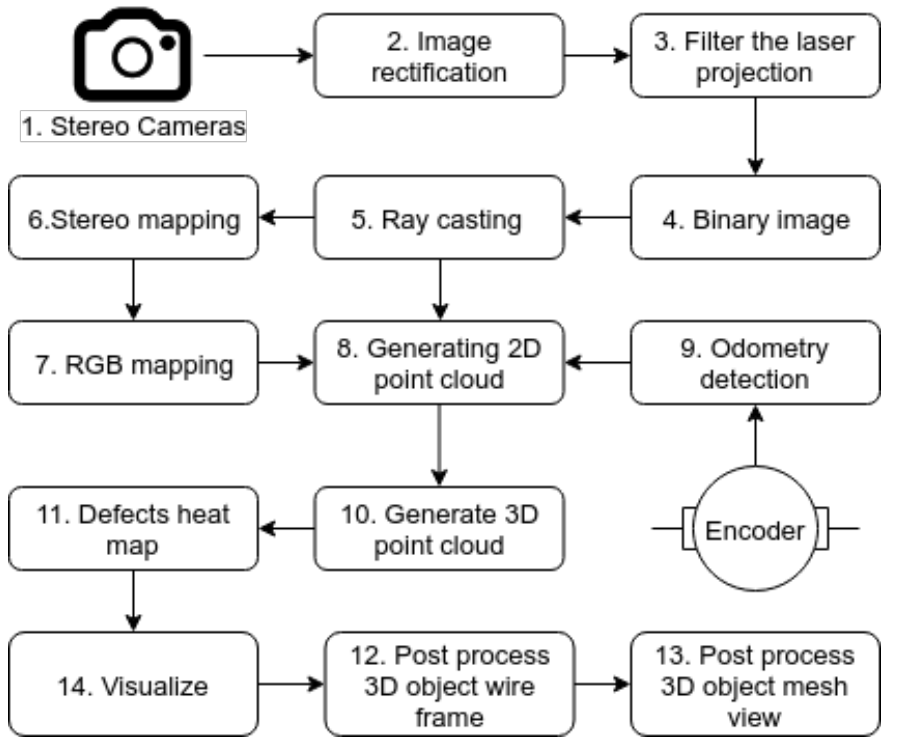

Fig. 2: Algorithm operation pipeline

can be projected on to the color image to obtain high quality RGB data of the pipe surface. In order to track the robot movements, wheel odometry has been used by fixing a rotary encoder with 1000 pulses/revolution on the robot. Further, to detect the orientation of the robot, stereo camera images were used. In order to run the whole system including image processing algorithms, a high-performance mother board has been incorporated. An Intel motherboard with $2.5 \mathrm{GHz}$ Quad core processor, 8GB RAM and 500GB SSD internal storage memory made the system run smooth in real-time. The whole system was built inside a water tight enclosure box to protect the electronics from water leaks.

\section{3D point cloud processing pipeline}

A set of standard image processing techniques were utilized to generate a dense high resolution point cloud in real-time. The execution pipeline of the algorithm is shown in Fig. 2.

1) Camera calibration: Image distortion is a common problem for any camera. Therefore, in order to get accurate measurements from the camera images, they generally need to be calibrated first using the equation (1) and (2), where $x$ and $y$ are the coordinates of the image pixel, $r$ is the radius from the image center to the pixel, and $k_{1}, k_{2}, k_{3}$ are the radial distortion coefficients that need to be determined through calibration.

$$
\begin{aligned}
& y_{\text {correction }}=y\left(1+k_{1} r^{2}+k_{2} r^{4}+k_{3} r^{6} \ldots+k_{n} r^{2 n}\right) \\
& x_{\text {correction }}=x\left(1+k_{1} r^{2}+k_{2} r^{4}+k_{3} r^{6} \ldots+k_{n} r^{2 n}\right)
\end{aligned}
$$

The image distortion factor changes when the pixel coordinates are far from the image center. Therefore, multiple levels of coefficients $\left(k_{1}, k_{2}, k_{3}\right)$ have to be calculated using training data. Most of the cameras satisfy the calibration by estimating the first three coefficients. 
When a 3D object is projected onto the camera 2D image plane, the relationship between the 3D coordinates and the 2D plane coordinates can be represented by the camera intrinsic and extrinsic parameters. Equation (3) defines the high level representation of the camera parameters. When $P_{w}$ is the 3D coordinates of the object and $P^{\prime}$ is the 2D coordinates of the object projected on the image plane, the camera calibration parameters are given by $K, R$ and $T$, where $K$ is intrinsic parameters and $R$ (rotation) and $T$ (translation) are extrinsic parameters.

$$
P^{\prime}=[K][R T] P_{w}
$$

Intrinsic parameters define the image focal length $\left(f_{x}, f_{y}\right)$, optical center $\left(c_{x}, c_{y}\right)$ and skew coefficients $(s)$ in pixels. Extrinsic parameters define the relationship of the camera translation $(T)$ and rotation $(R)$ within the world coordinates. In practical application of equation (3), it further breaks down into equation (4) where $X, Y, Z$ are the 3D world coordinates of the object and $x, y$ represent the coordinates of the object projected on the camera image plane.

$$
\left[\begin{array}{l}
x \\
y \\
1
\end{array}\right]=\left[\begin{array}{ccc}
f_{x} & s & c_{x} \\
0 & f_{y} & c_{y} \\
0 & 0 & 1
\end{array}\right]\left[\begin{array}{llll}
r_{11} & r_{12} & r_{13} & t_{1} \\
r_{21} & r_{22} & r_{23} & t_{2} \\
r_{31} & r_{32} & r_{33} & t_{3}
\end{array}\right]\left[\begin{array}{c}
X \\
Y \\
Z \\
1
\end{array}\right]
$$

Once the algorithm runs through a set of training data set (usually a checker board pattern with known dimensions) the coefficients can be identified. Those parameters can be used for sensor calibration to improve the accuracy of extracting pipe measurements.

2) Image masking and filtering: The binary mask of the laser circle is extracted from the calibrated image through thresholding. Occasionally, due to surface illumination variations the detected circle had varying line thicknesses, which adversely affected the center detection of the circle. Therefore, to refine this line, a unique filter which had subtract and bitwise-or image processing functions are implemented using OpenCV framework. The best fit parameters are determined by training the algorithm using different types of data sets obtained from different pipes under different lighting conditions.

3) Center detection and ray casting: The Hough transform algorithm is used to estimate the center of the pipe circle that is used for ray casting and generating a point cloud. The accuracy of the center detection has been much improved by the filter algorithm. By running the ray casting function, all the points related to the laser beam circle can be identified and saved into an array of vector points indexed by the angular rotation. The ray casting algorithm is used mainly to achieve 3 main goals. They are: to generate the point cloud by extracting laser points; stereo mapping to extract colour depth information and to generate a heat map for defect identification by calculating the distance between center to each point.

4) Stereo mapping: Stereo mapping algorithms are used to calculate the depth of each point generated from the ray casting algorithm. By using the generated stereo calibration parameters, ray casted points are projected on to the other
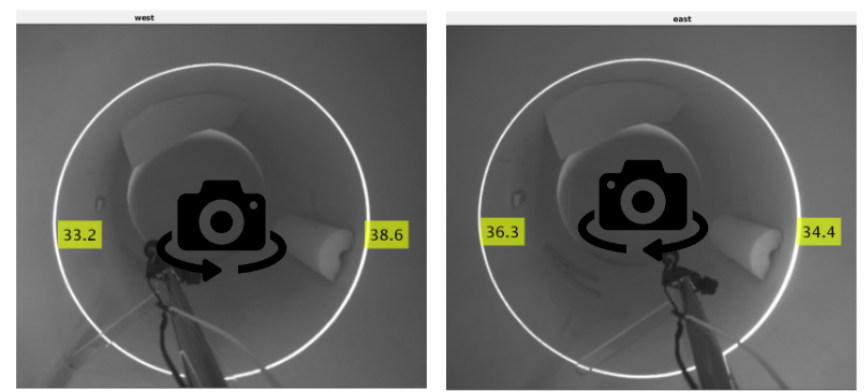

Fig. 3: Feature depth calculation for camera tilt detection

stereo camera image plane as epipolar lines. In rectified image plane, epipolar lines become parallel horizontal lines. Therefore stereo correspondence process becomes faster and efficient as the search can be done along the raws of the image. The corresponding point can be searched in the second image by detecting the locations where the laser beam cuts each epipolar line, which can be easily done by searching for highest intensity value. Usually two intersection points are detected due to the circular shape of the laser beam. Hence, in order to identify the correct match, the search algorithm can be updated to classify the points according to their location with respect to the center. Equations (5), (6) and (7) represent the high level calculations of the stereo mapping functionality.

When the disparity value taken as $d$, camera focal length defined as $f$, distance between two camera focal points defined as $T$, coordinates of the object position defined as $P=\left(X_{p}, Y_{p}, Z_{p}\right)$, object coordinates in the left camera image defined as $p_{l}=\left(x_{l}, y_{l}\right)$ and object coordinates in the right camera image defined as $p_{r}=\left(x_{r}, y_{r}\right)$; the relation between the coordinates can be defined as below;

$$
\begin{aligned}
& d=\left(x_{l}-x_{r}\right) \\
& x_{l}=\frac{X_{p} f}{Z p} ; x_{r}=\frac{\left(X_{p}-T\right) f}{Z p} ; y_{l}=y_{r}=\frac{Y_{p} f}{Z_{p}} ;
\end{aligned}
$$

From (5) and (6):

$$
Z_{p}=f \frac{T}{x_{l}-x_{r}}=f \frac{T}{d} ; X_{p}=x_{l} \frac{T}{d} ; Y_{p}=y_{l} \frac{T}{d}
$$

Once the depth information is available from the stereo mapping algorithm, it can be used to determine the orientation of the robot. If the robot tilt while moving forward the orientations are displayed on the system to maneuver it properly. An example depth calculation on two identified points are shown in Fig. 3 The depth information further can be used to show the pipe orientation in world coordinates. which is planned as a future enhancement.

5) $R G B$ mapping: The generated $3 \mathrm{D}$ points are used for RGB color mapping by projecting them on to the RGB image using the equation (8) and (9). When a 3D point $\left(P_{x}, P_{y}, P_{z}\right)$ 


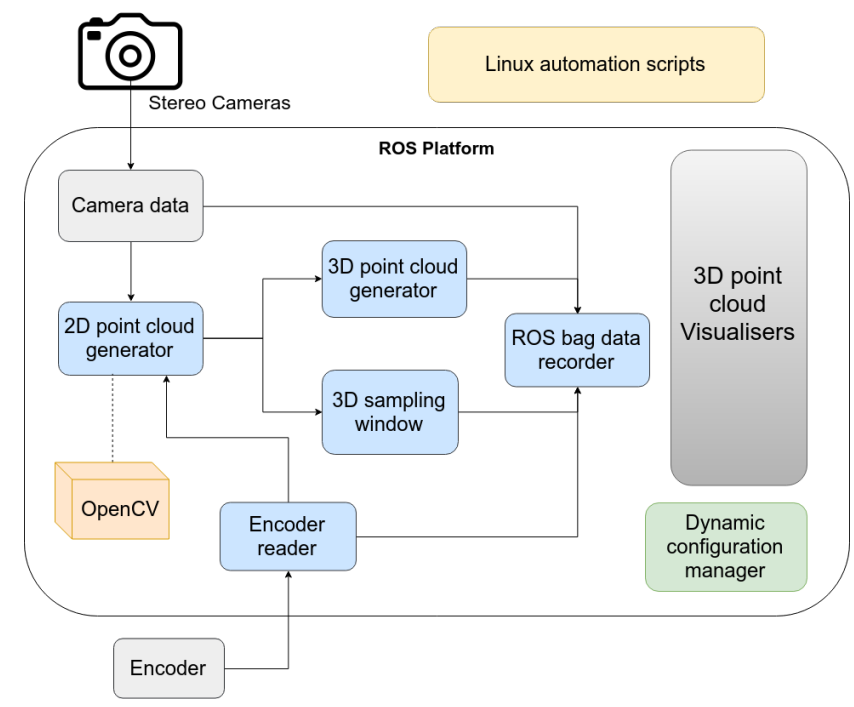

Fig. 4: Software architecture diagram

projects onto $2 \mathrm{D}$ point $\left(P_{x}^{\prime}, P_{y}^{\prime}\right)$ where $s$ is arbitrary scale factor and $c$ is arbitrary offset:

$$
\begin{aligned}
P_{x}^{\prime} & =s_{x} P_{x}+c_{x} \\
P_{y}^{\prime} & =s_{z} P_{z}+c_{z}
\end{aligned}
$$

Using matrix multiplication, this can be further broken into equation 10 .

$$
\left[\begin{array}{l}
P_{x}^{\prime} \\
P_{y}^{\prime}
\end{array}\right]=\left[\begin{array}{ccc}
s_{x} & 0 & 0 \\
0 & 0 & s_{z}
\end{array}\right]\left[\begin{array}{l}
P_{x} \\
P_{y} \\
P_{z}
\end{array}\right]+\left[\begin{array}{l}
c_{x} \\
c_{z}
\end{array}\right]
$$

By projecting the $3 \mathrm{D}$ point cloud on to the RGB camera image, the colour information of each point can be obtained. The projection parameters are estimated by stereo calibrating one of the IR cameras with the RGB camera.The color information is later fused with the point cloud data to generate RGB-D map of the pipe.

6) Software architecture: The full system was implemented on Robotic Operating System (ROS) framework using C++ and OpenCV libraries. In order to improve the real-time performances, the major functionalities are distributed into separate threads by creating appropriate ROS nodes. This approach enabled the full potential of parallel processing to enhance the performance. The software architecture is shown in Fig. 4. The images taken from the cameras are processed in the depth calculation node to generate the 3D point cloud of the laser ring w.r.t camera frame. As the robot moves forward, the odometry information is fused with the 3D point-cloud data of the laser rings to iteratively generate the 3D map of the pipe. By subscribing to each topic using ROS RVIZ like visualization system, the $3 \mathrm{D}$ profile map of the pipe can be seen in real-time. Linux automation scripts were employed to automate and start-up process with a single push button.

\section{EXPERIMENTS}

In order to experimentally validate the proposed 3D profiling system, a series of experiments were carried out on various pipe samples.

\section{A. Accuracy of the diameter measurements}

The ability of the proposed system to measure any ovality change with millimeter level of accuracy is important in applications where accurate defect quantification are needed. Therefore, the robotic laser profiling was done on a PVC pipe with a diameter of $300 \mathrm{~mm}$. The Root Mean Square Error (RMSE) was calculated across the circumference of the pipe for each sample period, and the test results are shown in Fig.5. The recorded RMSE values were always less than $1 \mathrm{~mm}$ proving the ability of the proposed system of measuring $300 \mathrm{~mm}$ pipe diameter with sub millimeter level of accuracy.

\section{B. Quantification of defects}

Next the pipe was modified with artificially planted defects for detection as shown in Fig. 8. The thickness of those artificially planted protruded defects were $3 \mathrm{~mm}$ or more and some were artificially made holes of $3 \mathrm{~mm}$ depth inside the pipe. After running the robotic laser profiling system across the pipe several times, RMSE values were calculated using the standard equation. The results are tabulated in Table I These test results show the ability of the proposed laser profiling system for quantifying both protruded and caved in defects with mm level of accuracy.

TABLE I: Test results

\begin{tabular}{|c|c|c|c|c|}
\hline \multirow{2}{*}{ Test } & \multirow{2}{*}{$\begin{array}{c}\text { Ground } \\
\text { truth }\end{array}$} & \multicolumn{2}{|c|}{ Result } & \multirow{2}{*}{ RMSE } \\
\cline { 3 - 4 } & & Minimum & Maximum & \\
\hline $\begin{array}{c}\text { PVC pipe } \\
\text { diameter }\end{array}$ & $300 \mathrm{~mm}$ & $299 \mathrm{~mm}$ & $301 \mathrm{~mm}$ & $\pm 1 \mathrm{~mm}$ \\
\hline $\begin{array}{c}\text { Artificial defect } \\
1 \text { (300mm pipe) }\end{array}$ & $3 \mathrm{~mm}$ & $2 \mathrm{~mm}$ & $4 \mathrm{~mm}$ & $\pm 1 \mathrm{~mm}$ \\
\hline $\begin{array}{c}\text { Artificial defect } \\
2 \text { (300mm pipe) }\end{array}$ & $10 \mathrm{~mm}$ & $9 \mathrm{~mm}$ & $11 \mathrm{~mm}$ & $\pm 1 \mathrm{~mm}$ \\
\hline $\begin{array}{c}\text { Artificial holes } \\
\text { (300mm pipe) }\end{array}$ & $3 \mathrm{~mm}$ & $2 \mathrm{~mm}$ & $4 \mathrm{~mm}$ & $\pm 1 \mathrm{~mm}$ \\
\hline
\end{tabular}

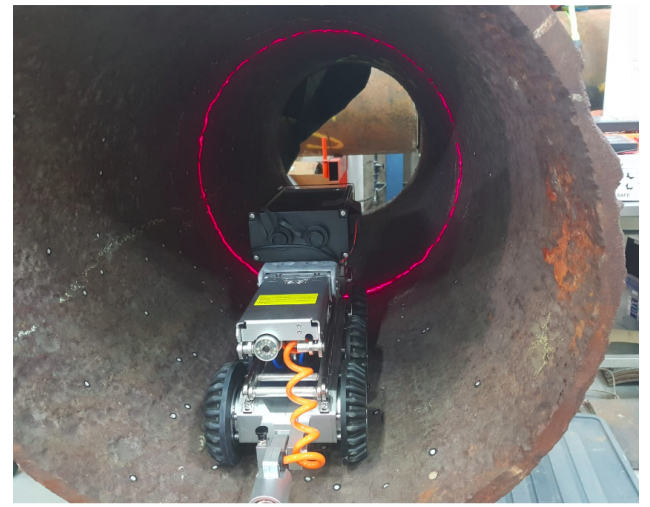

Fig. 7: The robot scanning a corroded metal pipe. Red laser projection has been used instead of the IR laser for image demonstration purposes. 


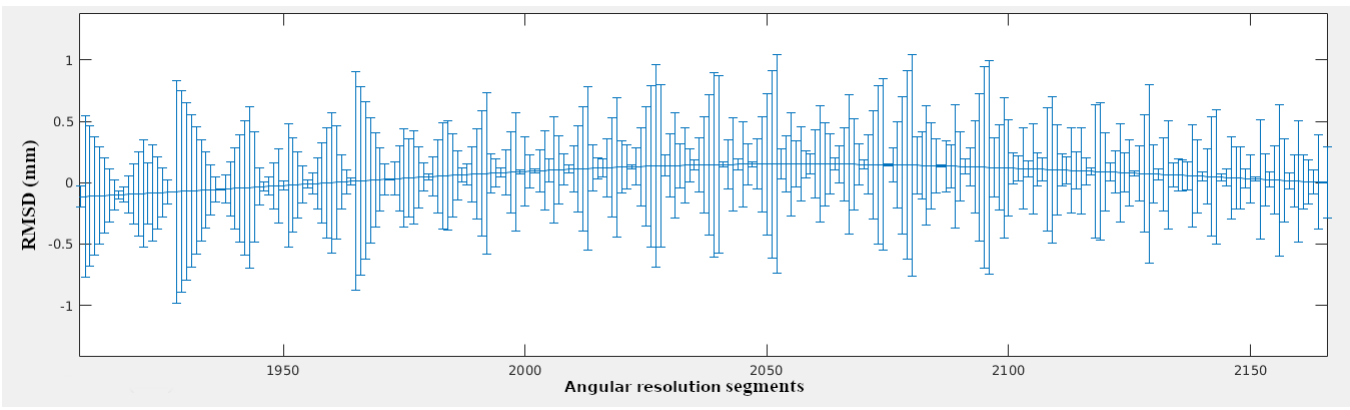

Fig. 5: Root mean square deviation from the ground truth

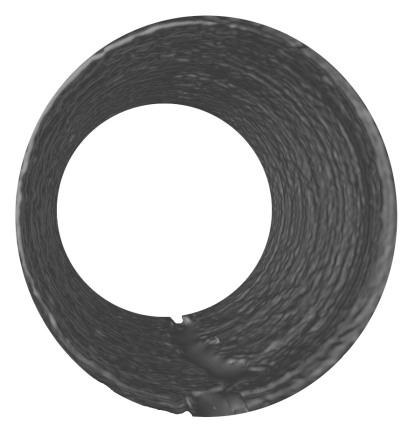

(a) 3D mesh of the pipe without colour

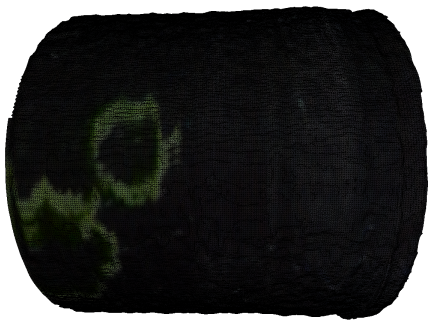

(d) 3D mesh view with embedded real colour

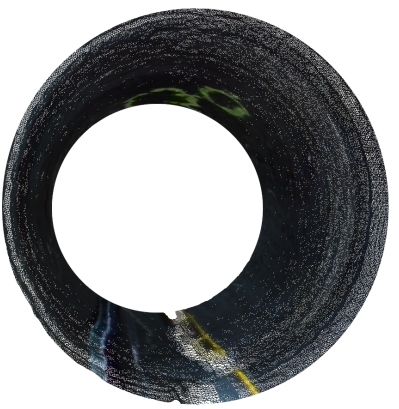

(b) 3D wire frame of the pipe

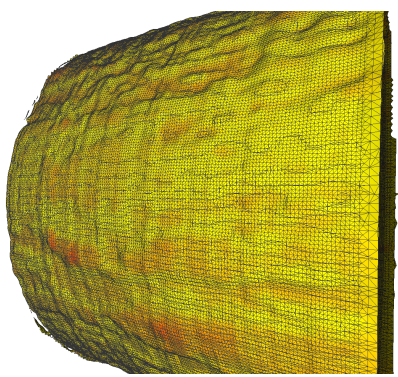

(e) Side view of the defects heat map

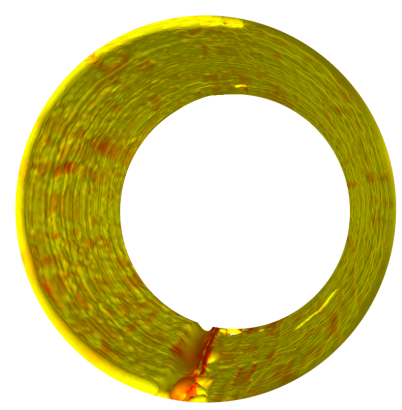

(c) Pipe 3D profile with defects heat map

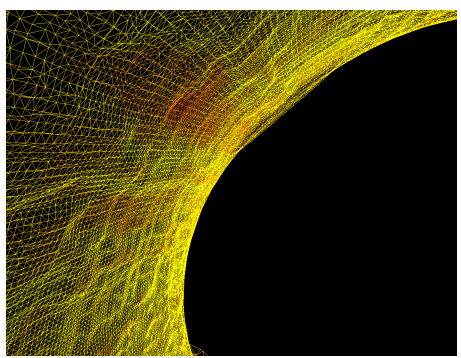

(f) Detailed mesh wire of the heat map

Fig. 6: 3D scan results

\section{Scanning corroded pipe samples}

After validating the sensors and testing the accuracy, several corroded pipe samples were used to test the entire system. Among them are corroded cast iron pipes with visible corrosion pits. Fig. 7 shows an example of performing a laser profiling in a corroded $600 \mathrm{~mm}$ metal pipe. The scan results are presented from Fig. 6a to Fig. 6f and the defects are highlighted and presented from Fig. 6c to Fig. 6f. Shades of red colour has been applied to the surface defects according to the corroded depth. The 3D profiling was able to map corrosion pits with an accuracy level of $2 \mathrm{~mm}$ for $600 \mathrm{~mm}$ pipe, which was verified through spot measurements. Fig. 6f] shows a magnified portion of the wire frame with highlighted corrosion pits and their depth heat-map. The proposed laser profiling system shows the ability to identify and quantify the depth of corrosion pits which can be a very useful parameter for carrying out maintenance. Fig. 6d shows the RGB-D colour map with visual information for qualitative assessment of

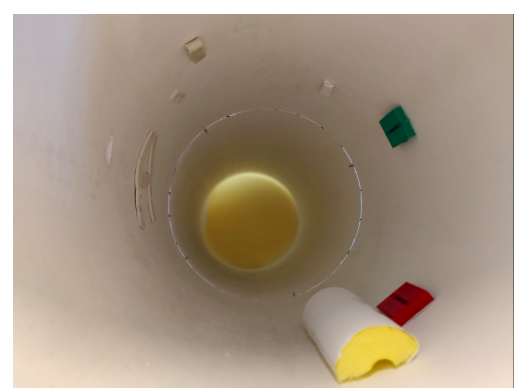

Fig. 8: Artificial defects placed on the pipe for accuracy tests

colour mapping. Fig. $6 \mathrm{~b}$ shows the wire mesh of the RGB colour mapped 3D mesh.

\section{Real-time performance}

Fig. 9 shows the real-time 3D point cloud generation in ROS RVIZ for a pipe with liner defects and joints. The 
hardware performance in combination with the optimized algorithms and parallel processing ROS nodes; the system is capable of processing the image frames without any lag (30 frames/second rate). In the prototype system with a $30 \mathrm{fps}$ camera the robot needs to travel $0.2 \mathrm{~m}$ per minute to acquire $1 \mathrm{~mm}$ accuracy along the pipe axis. Higher speeds contributes to lower accuracies however, wouldn't affect the real-time system performance. Table II presents system's performance for different point cloud resolutions.

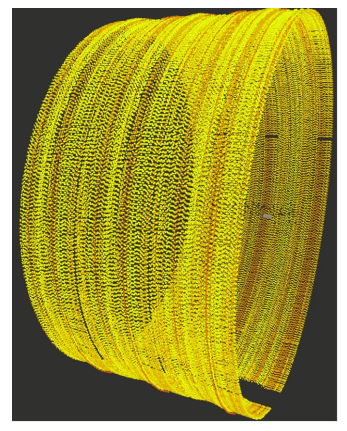

Fig. 9: A real-time visualization of the pipe scan in 3D

TABLE II: System performance

\begin{tabular}{|c|c|}
\hline Points per frame & Frame rate (fps) \\
\hline 1000 & 60 \\
\hline 3000 & 30 \\
\hline 6000 & 10 \\
\hline
\end{tabular}

\section{CONCLUSION}

In summary, this paper proposed a 3D laser profiling in pipelines using stereo vision and structured IR laser light. The performance and the accuracy of the proposed system have been improved considerably by employing image processing algorithms such as stereo mapping, ray casting, and RGB depth mapping in a processing pipeline. The system is capable of generating an accurate 3D point cloud of the internal surface of the pipe in real-time. The accuracy of the proposed system is validated on controlled pipe samples. Finally, the tests were carried out by scanning corroded pipe samples to identify defects. Further research is planned to improve the localization of the robot where it is planned to deploy on real pipeline to test its performance and accuracy.

\section{ACKNOWLEDGMENT}

This publication is an outcome from the project "Development of sensor suites and robotic deployment strategies for condition assessment of concrete sewer walls" funded by the Sydney Water Corporation.

\section{REFERENCES}

[1] K. Thiyagarajan, S. Kodagoda, R. Ranasinghe, D. Vitanage, and G. Iori, "Robust sensing suite for measuring temporal dynamics of surface temperature in sewers," Scientific Reports, vol. 8, no. 1, 2018.

[2] J. Valls Miro, N. Ulapane, L. Shi, D. Hunt, and M. Behrens, "Robotic pipeline wall thickness evaluation for dense nondestructive testing inspection," Journal of Field Robotics, vol. 35, no. 8, pp. 1293-1310, 2018.
[3] K. Thiyagarajan, "Robust Sensor Technologies Combined with Smart Predictive Analytics for Hostile Sewer Infrastructures," Ph.D. dissertation, University of Technology Sydney, 2018.

[4] K. Thiyagarajan, S. Kodagoda, L. V. Nguyen, and S. Wickramanayake, "Gaussian Markov Random Fields for Localizing Reinforcing Bars in Concrete Infrastructure," in 2018 Proceedings of the 35th International Symposium on Automation and Robotics in Construction, Berlin, 2018, pp. 1052-1058.

[5] K. Thiyagarajan, S. Kodagoda, and N. Ulapane, "Data-driven machine learning approach for predicting volumetric moisture content of concrete using resistance sensor measurements," in 2016 IEEE 11th Conference on Industrial Electronics and Applications, 2016, pp. 1288-1293.

[6] B. Li, X. Fan, J. Zhang, Y. Wang, F. Chen, S. Kodagoda, T. Wells, L. Vorreiter, D. Vitanage, G. Iori, D. Cunningham, and T. Chen, "Predictive Analytics Toolkit for H2S Estimation and Sewer Corrosion," in OZWater. Sydney: Australian Water Association, 2017.

[7] K. Thiyagarajan, S. Kodagoda, L. V. Nguyen, and R. Ranasinghe, "Sensor Failure Detection and Faulty Data Accommodation Approach for Instrumented Wastewater Infrastructures," IEEE Access, vol. 6, pp. 56562-56 574, 2018.

[8] K. Thiyagarajan, S. Kodagoda, and L. V. Nguyen, "Predictive analytics for detecting sensor failure using autoregressive integrated moving average model," in 2017 12th IEEE Conference on Industrial Electronics and Applications (ICIEA), 2017, pp. 1926-1931.

[9] K. Thiyagarajan, S. Kodagoda, and J. K. Alvarez, "An instrumentation system for smart monitoring of surface temperature," in 2016 14th International Conference on Control, Automation, Robotics and Vision (ICARCV), 2016, pp. 1-6.

[10] Z. Liu and D. Krys, "The use of laser range finder on a robotic platform for pipe inspection," Mechanical Systems and Signal Processing, vol. 31, pp. 246-257, 2012.

[11] J. Saenz, N. Elkmann, T. Stuerze, S. Kutzner, and H. Althoff, "Robotic systems for cleaning and inspection of large concrete pipes," in $20101 \mathrm{st}$ International Conference on Applied Robotics for the Power Industry, CARPI 2010, Fraunhofer IFF, 39106 Magdeburg, Germany, 2010.

[12] O. Duran, K. Althoefer, and L. D. Seneviratne, "Automated pipe defect detection and categorization using caniera/laser-based profiler and artificial neural network," IEEE Transactions on Automation Science and Engineering, vol. 4, no. 1, pp. 118-126, 2007.

[13] J.-S. Yoon, M. Sagong, J. S. Lee, and K.-s. Lee, "Feature extraction of a concrete tunnel liner from 3D laser scanning data," $N D T$ and $E$ International, vol. 42, no. 2, pp. 97-105, 2009.

[14] M. Nasrollahi, N. Bolourian, Z. Zhu, and A. Hammad, "Designing LiDAR-equipped UAV platform for structural inspection," in 35th International Symposium on Automation and Robotics in Construction and International AEC/FM Hackathon: The Future of Building Things, ISARC 2018. Department of Building, Civil and Environmental Engineer, Concordia University, Canada: International Association for Automation and Robotics in Construction I.A.A.R.C), 2018.

[15] E. Ujkani, J. Dybedal, A. Aalerud, K. B. Kaldestad, and G. Hovland, "Visual Marker Guided Point Cloud Registration in a Large Multi-Sensor Industrial Robot Cell," in 14th IEEE/ASME International Conference on Mechatronic and Embedded Systems and Applications, MESA 2018. Department of Engineering Sciences, Mechatronics Group, University of Agder, Norway: Institute of Electrical and Electronics Engineers Inc., 2018.

[16] N. Stanić, M. Lepot, M. Catieau, J. Langeveld, and F. Clemens, "A technology for sewer pipe inspection (part 1): Design, calibration, corrections and potential application of a laser profiler," Automation in Construction, vol. 75, pp. 91-107, 2017.

[17] M. Lepot, N. Stanić, and F. H. L. R. Clemens, "A technology for sewer pipe inspection (Part 2): Experimental assessment of a new laser profiler for sewer defect detection and quantification," Automation in Construction, vol. 73, pp. 1-11, 2017.

[18] R. Rantoson, C. Stolz, D. Fofi, and F. Mériaudeau, "Non contact 3D measurement scheme for transparent objects using UV structured light," in 2010 20th International Conference on Pattern Recognition, ICPR 2010, Laboratoire Le2i-CNRS UMR 5158, Université de Bourgogne, 12, Rue de la Fonderie, 71200 Le Creusot, France, 2010, pp. 16461649.

[19] J. Kofman, J. T. Wu, and K. Borribanbunpotkat, "Multiple-line fullfield laser-camera range sensor," in Optomechatronic Computer-Vision Systems II, vol. 6718, Dept. Systems Design Engineering, University of Waterloo, Waterloo, ON N2L 3G1, Canada, 2007. 